\title{
MEG alpha activity decrease reflects destabilization of multistable percepts
}

\author{
Daniel Strüber ${ }^{\mathrm{a}}$, Christoph S. Herrmann ${ }^{\mathrm{b}, *}$ \\ ${ }^{a}$ Institute of Psychology and Cognition Research \& Center for Cognitive Sciences, University of Bremen, P.O. Box 330 440, 28334 Bremen, Germany \\ ${ }^{\mathrm{b}}$ Max-Planck-Institute of Cognitive Neuroscience, P.O. Box 500 355, 04303 Leipzig, Germany
}

Accepted 27 March 2002

\begin{abstract}
Multistable stimuli offer the possibility to investigate visual awareness, since they evoke spontaneous alternations between different perceptual interpretations of the same stimulus and, therefore, allow to dissociate perceptual from stimulus-driven mechanisms. In the present study, we used an ambiguous motion paradigm and compared endogenous reversals of perceived motion direction which occur spontaneously during constant ambiguous stimulation with exogenous reversals that were induced externally by changes of stimulation. Contrasting the two conditions allowed to investigate processes that trigger endogenous reversals, since the related activity should be absent in the exogenous reversal condition. We employed ambiguous dot patterns which can easily be transformed to present two stable motion directions in order to induce exogenous pattern reversals. Whole-head MEG was recorded from 10 subjects. We analyzed event-related fields (ERFs) and oscillatory activity in the alpha and gamma ranges. The results showed P300-like slow waves in response to both endogenous and exogenous reversals reflecting the conscious recognition of pattern reversals. Analyses in the gamma-band did not reveal any significant modulations. The alpha activity showed different time courses for endogenous and exogenous reversals. While the exogenous alpha activity decreased in temporal relation to the pattern reversal, the endogenous alpha activity displayed a continuous decrease starting in the time interval preceding the reversal. This time course of the endogenous alpha activity is consistent with a bottom-up approach to figure reversals, since it reflects a process of destabilization of the actual percept until a switch of visual awareness occurs.
\end{abstract}

(C) 2002 Elsevier Science B.V. All rights reserved.

Theme: Neural basis of behaviour

Topic: Cognition

Keywords: Alpha-band; Ambiguous pattern; Multistable perception; Visual awareness; Event-related fields; Magnetoencephalography

\section{Introduction}

After decades of actively ignoring the problem of consciousness, scientists of many persuasions are finally beginning to work on it. Especially the search for neural correlates of consciousness has received considerable interest, since modern neurobiological techniques offer a new perspective on this issue for cognitive neuroscience. Visual awareness is considered to be a favorable form of consciousness to study neurobiologically [11], and has

\footnotetext{
*Corresponding author. Tel.: +49-341-994-0250; fax: +49-341-9940204; http://www.csherrmann.de.

E-mail addresses: struber@uni-bremen.de (D. Strüber),
} herrmann@cns.mpg.de (C.S. Herrmann). been intensively studied recently [40,55]. The most promising approach to date in studying visual awareness has been to use experimental paradigms in which physical stimulation and behavior are held constant, but perception changes [54]. Exactly this is the case with so-called ambiguous or multistable stimuli, since they induce spontaneous alternations between different perceptual interpretations of the same stimulus and, therefore, allow to dissociate perceptual from stimulus-driven mechanisms.

Multistable visual phenomena consist of at least two types. One type of multistability is represented by classical ambiguous figures like the well-known Necker cube, Rubin's vase/faces or Jastrow's duck/rabbit figure. While these patterns evoke bistable perception during normal binocular viewing, the other type of multistability origi- 
nates from presenting the two eyes with incongruent stimuli leading to binocular rivalry, such that perception alternates between each monocular view.

During the past decade, most of the studies using multistable patterns to investigate visual awareness have concerned the phenomenon of binocular rivalry. An intensive research was conducted with a variety of methods including single-cell electrophysiology $[14,37,38,42,57,59]$, electro- and magnetoencephalography (EEG/MEG) [10,25,61,71,73], neuroimaging [44,45,53,70,69], and psychophysical studies $[8,16,33,36,41]$. A recent review on this issue is given by Blake and Logothetis [7]. Most of these rivalry studies were more or less directly concerned with the question, at what level of visual processing rivalry 'occurs'. Accordingly, the above mentioned neurophysiological studies analyzed neuronal activity that follows the changing percepts rather than the constant retinal inputs.

However, another interesting issue to study with multistable patterns is the neuronal mechanism which triggers the spontaneous fluctuations in awareness. For this kind of analysis, one has to focus on activity that precedes the perceptual transitions, rather than on activity associated with each of the perceptual states. Functional magnetic resonance imaging (fMRI) was used to investigate brain activity related to the subjects' perceptual alternations during binocular rivalry and during a control condition of non-rivalrous perceptual changes [44]. Greater activation during rivalrous perceptual transitions compared to the control condition was found in extrastriate visual area 19 and frontoparietal regions of the right hemisphere. Using classical ambiguous figures, a relation between eventrelated hemodynamic activity and perceptual reversals was provided by another fMRI study [27]. This study compared perceptual transition-related fMRI signals with those obtained during periods of perceptual stability within the same experimental session. Transiently increased activity during perceptual reversals was observed bilaterally in ventral occipital, intraparietal, and some frontal areas. These increases of activity were accompanied with transient deactivations in primary visual cortex and the posterior thalamus. The authors concluded that the distribution of activations during perceptual reversals reflect an intimate linkage of perceptual and attentional processes in the dynamic of metastable visual awareness, whereas the deactivations in primary visual cortex and thalamus could reflect a functional contribution to perceptual stability and the temporary absence of a clear-cut winner in the rivalry between two percepts, respectively.

Although these fMRI studies have been extremely useful in identifying the brain regions that are activated or deactivated during perceptual reversals, information about the temporal dynamics underlying multistable perception remained limited due to the relatively low temporal resolution of fMRI measures. Therefore, it is worth investigating the time course of brain activity associated with perceptual alternations by means of EEG or MEG providing high temporal resolution. In an EEG study, gamma-band activity $(30-50 \mathrm{~Hz})$ was analyzed during unstable (reversal phase) and stable (non-reversal phase) perceptual states while subjects continuously observed an ambiguous motion paradigm [4]. The results demonstrated enhanced gamma-band activity compared to spontaneous EEG, especially over right frontal areas during the reversal phase, indicating a functional role for gamma activity in figure reversals. More recently, the time course of the standard EEG frequency bands was measured during perceptual reversals between motion directions using the same ambiguous motion paradigm as in the study mentioned above [46]. This study showed that perceptual alternations were preceded by a decrease of EEG frequencies (reflecting a vigilance decrease), and followed by a frequency increase (reflecting an arousal reaction). The frequency decrease before the reversal was due to decreased activity in the theta- and alpha-bands. Recently, alpha activity decrease in EEG has been reported also during perceptual reversals of the Necker cube [23]. As demonstrated by these EEG studies, the alpha and gamma frequency bands seem to be functionally related to figure reversals.

The main purpose of the present study was to define more specifically the functional roles of the alpha- and gamma-bands during perceptual alternations with ambiguous figures. We used MEG recordings of healthy subjects while they observed a reversible motion paradigm based on apparent motion. We compared endogenous reversals which occur spontaneously during constant ambiguous stimulation with exogenous reversals that were induced externally by changes of stimulation. Contrasting the two conditions allowed us to investigate processes that trigger endogenous reversals, since the related activity should be absent in the exogenous reversal condition. According to the above mentioned EEG studies on alpha activity $[23,46]$, we hypothesized that a decrease of alpha activity preceding perceptual reversals reflects a mechanism of destabilizing the actual percept which is specific for endogenous pattern reversals. This alpha activity decrease might be accompanied by an increase of gamma activity [4]. We further hypothesized the occurrence of transient gamma activity during both endogenous and exogenous pattern reversals since the transition from one conscious experienced percept to the other is related to the binding of a new percept which is known to be mediated by gamma oscillations [26,68]. Therefore, it should make no difference whether the binding of the other motion direction is induced by endogenous or externally induced perceptual reversals. Similarly, it has been shown previously that gamma activity does not differentiate between feature binding of real or illusory triangles [67].

In addition to analysing the alpha and gamma activity, we also measured event-related fields (ERFs) in response to the endogenous and exogenous pattern reversals. In 
previous EEG experiments, it could be shown that endogenous pattern reversals induce a slow positive wave while continuously viewing an ambiguous motion display [5,64]. Therefore, the occurrence of similar components was expected also for the MEG recordings of the present study. A comparison of the ERFs in response to endogenous reversals and externally generated pattern reversals allowed us to estimate the time of reversal which determines the relevant time interval to search for alpha and gamma activity reflecting the initiation of a reversal.

\section{Methods}

\subsection{Subjects}

Ten subjects with a mean age of 22.3 years (ranging from 18 to 24 years, four female) participated in the study. One subject had to be excluded from alpha analysis due to excessive alpha activity probably caused by low vigilance. All subjects were right-handed (laterality index 90-100 according to the Edinburgh handedness test) and had normal or corrected-to-normal vision. They showed no signs of neurological or psychiatric disorders and all gave written informed consent.

\subsection{Stimuli}

Fig. 1 shows the stimuli used to generate the endogenous and exogenous changes of apparent motion directions. Presenting stimuli A1 and A2 successively resulted in the perception of apparent motion of two dots either in horizontal or in vertical direction. During prolonged observation of this display with sight fixation spontaneous reversals between vertical and horizontal motion directions occur-a phenomenon known as 'stroboscopic alternative motion', which has been studied frequently in the context of EEG and multistable perception [4,5,46,62,64]. To induce an exogenous pattern reversal between vertical and horizontal motion, the spatial arrangement of the dots was modified. Successive presentation of stimuli HL and HR in Fig. 1 led to the perceptually stable impression of horizontal motion, while stimuli VT and VB produced unambigu- ous vertical motion. Changing the presentation between the two unambiguous patterns HL/HR and VT/VB led to an externally induced (exogenous) reversal of perceived motion direction that closely mimics the spatial/temporal conditions of the ambiguous change of motion direction.

Each dot subtended a visual angle of $0.23^{\circ}(4 \mathrm{~mm})$ and their horizontal and vertical distance was $1.72^{\circ}$ visual angle $(3 \mathrm{~cm})$. Dots were presented in white on a black background. The screen was placed $1 \mathrm{~m}$ in front of the subjects. For the ambiguous motion display, the stimuli A1 and A2 were presented successively for $165 \mathrm{~ms}$ each with an inter-stimulus-interval (ISI) of $85 \mathrm{~ms}$. Thus, each single stimulus (A1 vs. A2) was shown every $500 \mathrm{~ms}$, leading to a repetition rate of $2 \mathrm{~Hz}$ during continuous presentation. The same timing was used for the unambiguous horizontal (HL, HR) and vertical (VT, VB) motion display.

\subsection{Experimental procedure}

The experiment was performed in four sessions. Session 1 served as a training session in order to make the subjects familiar with the ambiguous motion display and to obtain their individual reversal rates. MEG was not recorded. Stimuli A1 and A2 were presented successively. In sessions 2 and 3, MEG measurements were performed using the exogenous pattern reversals HL and HR for horizontal and VT and VB for vertical motion, respectively. Subjects were instructed to either count silently (session 2) or press a button (session 3 ) in order to reveal the influence of motor activity on ERFs. Furthermore, the ERFs in response to the exogenous pattern reversals obtained in sessions 2 and 3 were used to estimate the time of reversal for the ambiguous pattern, which was presented in session 4 where A1 and A2 were again presented. A more detailed description of the sessions is given below. The order of sessions was kept constant across subjects.

During all sessions, subjects were instructed to fixate a little cross in the center of each display.

\subsubsection{Session 1}

In session 1, subjects perceived ambiguous changes in motion direction and were instructed to silently count how often a change of perceived motion direction occurred.

Ambiguous motion:

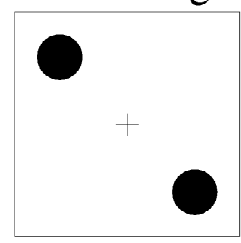

A1

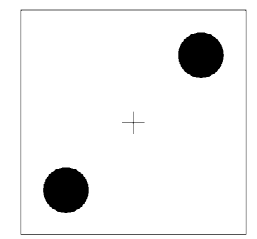

A2

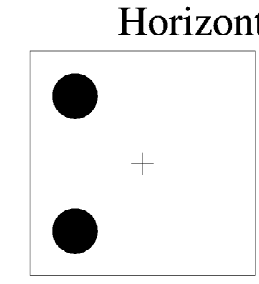

$\mathrm{HL}$

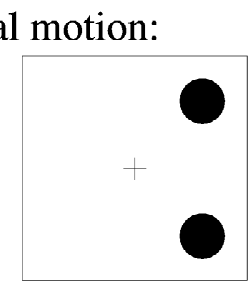

HR

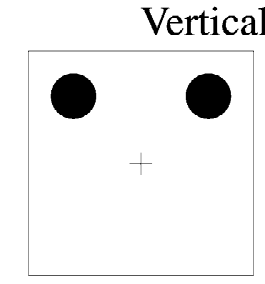

VT

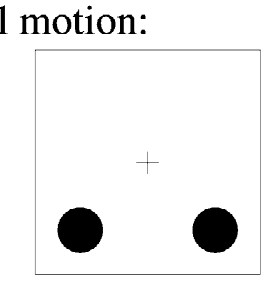

VB

Fig. 1. Ambiguous motion perception was induced by alternating the two stimuli A1 and A2. The unambiguous horizontal motion was generated by an alternating presentation of the two stimuli horizontal-left (HL) and horizontal-right (HR). Alternation of vertical-top (VT) and vertical-bottom (VB) produces unambiguous vertical motion. ' + ' served as fixation mark. 
Session 1 lasted $5 \mathrm{~min}$. This session of the experiment was intended to reveal the individual reversal rate of each subject in order to subsequently adjust the number of pattern reversals in the exogenous sessions. This procedure is required, because the reversal rate varies remarkably across subjects $[9,46]$. Accordingly, the individual ISI and the temporal probability of the critical event (reversal) differ across subjects which is known to affect cognitive brain potentials in classical 'oddball' studies [12,13,52,65]. Additionally, it was shown recently that the reversal rate is a critical variable for the amount of gamma activity during multistable perception $[62,63]$. Thus, ISI and probability of reversals should be kept approximately constant across sessions for each subject (as described in the next section).

\subsubsection{Session 2}

In session 2, subjects viewed the externally induced pattern reversals between vertical and horizontal motion directions during $10 \mathrm{~min}$ of presentation time. They were instructed to silently count the changes of motion direction in order to rule out the effects of motor activity. The number of exogenously generated pattern reversals was adapted from the perceived endogenous changes in motion direction from session 1. In order to generate exogenously determined reversals that correspond to the individually varying reversal rates of session 1 , we computed 10 different stimulation sequences with mean reversal rates of 5.0, 5.2, 5.8, 6.4, 7.4, 9.0, 10.4, 12.4, 16.0 and 20.2 reversals per minute. The time intervals between changes of motion direction were randomized. Each subject was exposed to a sequence of exogenous pattern reversals which corresponded best to their individual reversal rate, as obtained in session 1 .

\subsubsection{Session 3}

In session 3, the identical stimulus presentation as in session 2 was repeated, but subjects were asked to press a button whenever a change of perceived motion direction occurred. Half of the subjects were instructed to press a button with their left index finger when the perceived motion direction changed from horizontal to vertical and with their right index finger in the opposite case.

The other half of the subjects was instructed oppositely. The contrast of the effects in sessions 2 and 3 offers the possibility to control the influence of motor activity related to the button press.

\subsubsection{Session 4}

Stimuli were identical to those in session 1 , but subjects were instructed to signal every reversal of perceived motion direction with a button press since the endogenously induced reversals cannot be observed directly. The assignment of hand of response to perceived change of motion direction was identical to session 3. This session lasted $15 \mathrm{~min}$. The comparison of sessions 3 and 4 was intended to reveal differences in brain activity between stimulus-driven (exogenous) and self-generated (endogenous) pattern reversals (see Table 1 for an overview).

\subsection{Data acquisition}

MEG was recorded continuously with a BTI 148 channel whole-head system (MAGNES WHS 2500). MEG data were sampled at $678.168 \mathrm{~Hz}$ (on-line $0.1 \mathrm{~Hz}$ analog high-pass and $200 \mathrm{~Hz}$ low-pass filtering). Horizontal and vertical EOG was registered with four additional EEG electrodes.

Subjects' head positions were recorded via five electric coils before and after each session to control for head movements. If any of the coils coordinates $(x, y$, or $z$ ) moved more than $5 \mathrm{~mm}$, the recording was repeated and data of that session were rejected.

\subsection{Data analysis}

\subsubsection{Rejection of artefacts}

All MEG epochs were first automatically and then manually inspected for artefacts and rejected if eye-movement artefacts or sensor drifts were detected. For automatic detection, we computed the standard deviation in a moving time window and epochs were rejected if a threshold was exceeded. EOG electrodes and MEG channels were checked with thresholds of $30 \mu \mathrm{V}$ and $1100 \mathrm{fT}$, respectively. Window sizes were $200 \mathrm{~ms}$ for EOG and $3 \mathrm{~s}$ for MEG.

\subsubsection{ERFs (stimulus-locked vs. response-locked averaging)}

In session 2, subjects were instructed to silently count the exogenous changes of motion direction. MEG epochs were computed stimulus-locked from $100 \mathrm{~ms}$ prior to the exogenous reversal of motion direction until $700 \mathrm{~ms}$ after (forward-averaging). Baselines were computed for the time interval $500 \mathrm{~ms}$ prior to the exogenous reversal where a constant motion direction was perceived.

Table 1

Overview of sessions and investigated effects

\begin{tabular}{lll}
\hline Sessions & Pattern reversal & Investigated effect \\
\hline 1 & Endogenous & Individual reversal rates \\
2 vs. 3 & Exogenous & Motor activity \\
3 vs. 4 & Exogenous vs. endogenous & Activity specific for endogenous reversals
\end{tabular}


In session 3 , subjects were instructed to press a button in response to the exogenous pattern reversals. Therefore, both stimulus-locked and response-locked averaging was possible. For a comparison with session 2, averaging epochs and baselines were computed in the same manner as described for session 2, i.e., stimulus-locked (forwardaveraging), while the data were averaged response-locked to the button-press (backward-averaging) for a comparison with session 4. Furthermore, session 3 allowed a comparison between stimulus-locked and response-locked averaging of the same data set. Evaluating the influence of response-locked averaging on the ERF morphology is important for a comparison with the ERFs obtained in session 4, because backward-averaging with the button press as reference creates a remarkable jitter due to subjects' varying reaction times [22].

In session 4 , the perceived change of motion direction is generated endogenously, i.e., no external event indicates the exact time of a perceived change. Therefore, a response-locked analysis of the time interval preceding the button response (backward-averaging) was applied in order to analyse the reversal related activity. Averaging epochs lasted from $1000 \mathrm{~ms}$ before the button press until $500 \mathrm{~ms}$ after. Baselines were computed for the time interval of 500 ms before these epochs, i.e., from $-1500 \mathrm{~ms}$ until -1000 ms with respect to the button press. The reported baselines were computed and subtracted prior to computing the averaged ERFs. All ERFs are displayed after $25 \mathrm{~Hz}$ lowpass filtering to reduce noise in the signals.

\subsubsection{ERFs (estimating the time of reversal)}

As mentioned above, the endogenous pattern reversals can only be observed indirectly by the subjects' motor response. In the present study, we were able to estimate the time of the endogenous reversals by a comparison with the exogenous pattern reversal conditions. One possibility is to take the mean reaction time (RT) obtained in session 3 as the estimated time interval between endogenous reversal and button press in session 4. However, as a more direct measure of this time interval, we used the mean peak latencies of the ERFs in response to the externally generated pattern reversals. Since the exogenous pattern reversals of session 3 allowed precise stimulus-locked averaging as well as response-locked averaging, we chose the ERFlatencies of this session to estimate the time of reversal in session 4.

\subsubsection{Oscillatory activity}

In order to compute the total oscillatory activity (the sum of evoked and induced activity) for individual frequencies, we applied a wavelet-based time-frequency analysis [18,20].

The time intervals in which the alpha activity was analyzed lasted from $2000 \mathrm{~ms}$ before the button press until
$250 \mathrm{~ms}$ after, i.e., response-locked analyses were performed. This long interval of $2000 \mathrm{~ms}$ prior to the button press was chosen in order to include possible processes of perceptual destabilization prior to the reversal which were hypothesized to occur in the alpha-band. The gamma-band was analyzed in a shorter time interval of -1000 to $0 \mathrm{~ms}$ (button press) during which binding-related activity was expected.

\subsubsection{Statistical analysis}

In order to avoid a loss of statistical power that is inherent when repeated measures analyses of variance (ANOVAs) are used to quantify multi-channel physiological recordings [48], sensors were pooled to four topographical regions of interest (ROIs). The regions included the following sensors (cf. Fig. 2): left anterior region (LAR): A4, A5, A6, A15, A16, A17, A18, A31, A32, A33, A34, A35, A51, A52, A53, A54, A55, A72, A73, A74, A75, A76, A77, A95, A96, A97, A113, A114, A115, A131, A132. Left posterior region (LPR): A7, A8, A19, A20, A21, A36, A37, A38, A39, A56, A57, A58, A59, A60, A78, A79, A80, A81, A82, A98, A99, A100, A101, A102, A103, A116, A117, A118, A119, A120, A121, A133, A134. Regions over the right hemisphere included the homologous electrodes. For statistical analyses root mean square (RMS) values of ERF amplitudes were pooled across the sensors in each of the ROIs. The use of RMS values avoids effects due to the bipolar fields generated by each dipole which is inherent in MEG measurements.

All effects with more than two degrees of freedom in the numerator were adjusted for violations of sphericity which are inherent in repeated-measures analyses and Greenhouse-Geisser epsilons were used to compute the $P$ values [15].

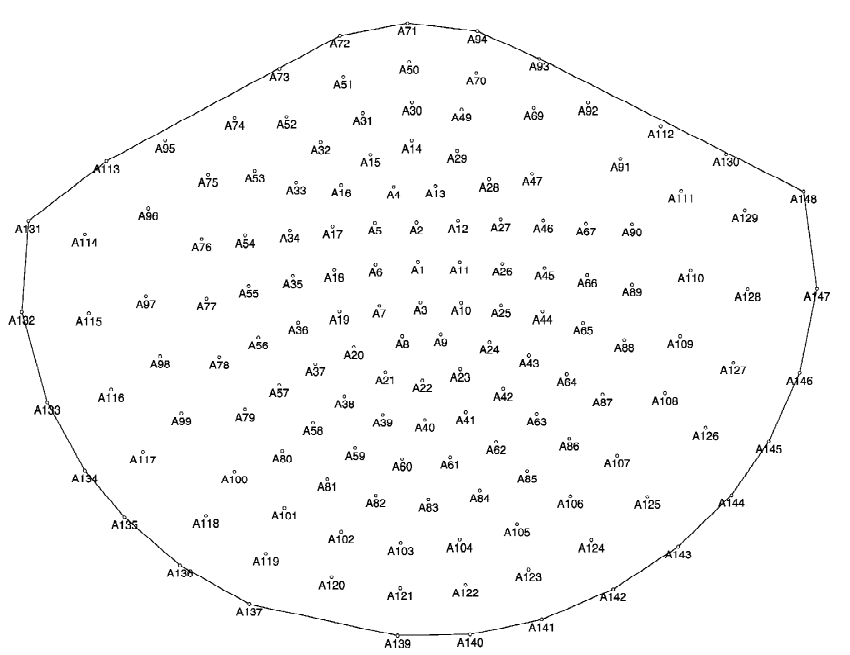

Fig. 2. Location of the 148 sensors as viewed from above (nose at top). 
Table 2

Individual reversal rates in reversals per minute

\begin{tabular}{lcccr}
\hline Subject & Session 1 & Session 2 & Session 3 & Session 4 \\
\hline 1 & $10.8(10.4)$ & $10.4(0 \%)$ & $9.7(6.7 \%)$ & 8.7 \\
2 & $9.0(9.0)$ & $8.8(2.2 \%)$ & $8.8(2.2 \%)$ & 6.4 \\
3 & $11.6(12.4)$ & $13.6(9.6 \%)$ & $12.3(0.1 \%)$ & 11.1 \\
4 & $9.4(9.0)$ & $10.2(13.3 \%)$ & $10.3(14.4 \%)$ & 7.3 \\
5 & $7.4(7.4)$ & $8.2(10.8 \%)$ & $7.3(1.4 \%)$ & 10.7 \\
6 & $12.0(12.4)$ & $12.3(0.1 \%)$ & $12.5(0.1 \%)$ & 9.7 \\
7 & $5.6(5.8)$ & $5.1(12.1 \%)$ & $6.1(5.2 \%)$ & 9.3 \\
8 & $13.0(12.4)$ & $12.3(0.1 \%)$ & $12.3(0.1 \%)$ & 18.1 \\
9 & $4.6(5.0)$ & $4.8(4 \%)$ & $5.0(0 \%)$ & 5.2 \\
10 & $1.0(5.0)$ & $4.8(4 \%)$ & $4.9(2 \%)$ & 2.3 \\
\multirow{2}{*}{ Mean } & $8.4(8.9)$ & $9.0(5.6 \%)$ & $8.9(3.2 \%)$ & 8.9 \\
\hline
\end{tabular}

For session 1, the corresponding exogenous stimulation sequences as used for sessions 2 and 3 are given in parentheses. For sessions 2 and 3 the percentual errors are given in parentheses.

\section{Results}

\subsection{Behavioral results}

Table 2 displays the individual reversal rates of the 10 subjects in reversals per minute obtained with the ambiguous (sessions 1,4) and unambiguous (sessions 2, 3) patterns. For session 1, the exogenously generated stimulus sequences which corresponded best to the individual reversal rates are given in parentheses (see Section 2.3.2). These stimulus sequences were subsequently presented in sessions 2 and 3 for which the percentual errors are indicated in parentheses as computed from the difference of presented to perceived reversals. Since in session 2 the subjects silently counted the exogenous pattern reversals, false positives and misses could not be differentiated. For session 3, false positives and misses were collapsed. More errors were made in the silent-counting (session 2) than button-pressing task (session 3). As can be seen from session 4 in Table 2, the reversal rates differed remarkably across subjects. The range of reversals per min in session 4 was between 2.3 (subject 10) and 18.1 (subject 8) with an overall mean of 8.9 reversals per minute. This corresponds to a range of mean reversal times per minute (i.e., mean time intervals between reversals) from $26.1 \mathrm{~s}$ to $3.3 \mathrm{~s}$ with an overall mean reversal time of $9 \mathrm{~s}$. Despite the overall variability of the reversal rate, the procedure of fitting the individual reversal rates for endogenous and exogenous changes in motion direction was successful, as indicated by the relatively constant reversal rates for each subject across sessions in Table 2 .

Table 3 displays the RTs of the 10 subjects separated by hand of response as obtained in session 3. The overall mean RT was 550.6 $\pm 68.5 \mathrm{~ms}$.

An ANOVA with factors hand (left, right) found no significant differences between response times of the left (559 $\mathrm{ms}$ ) and right hand (542 ms): $F(1,9)=0.67, P=0.43$. A further ANOVA with the factor subject (10 subjects)
Table 3

Reaction times (RTs) for reporting the perceived reversals of motion direction in session 3 with the left and right hand, respectively

\begin{tabular}{llll}
\hline Subject & $\mathrm{H} \Rightarrow \mathrm{V}$ & RT left $(\mathrm{ms})$ & RT right $(\mathrm{ms})$ \\
\hline 1 & $\mathrm{R}$ & 465 & 466 \\
2 & $\mathrm{R}$ & 646 & 559 \\
3 & $\mathrm{~L}$ & 447 & 430 \\
4 & $\mathrm{~L}$ & 587 & 627 \\
5 & $\mathrm{~L}$ & 525 & 486 \\
6 & $\mathrm{R}$ & 533 & 573 \\
7 & $\mathrm{R}$ & 663 & 514 \\
8 & $\mathrm{R}$ & 535 & 540 \\
9 & $\mathrm{~L}$ & 606 & 668 \\
10 & $\mathrm{~L}$ & 581 & 560 \\
& & & 542 \\
\hline
\end{tabular}

An ' $\mathrm{R}$ ' or ' $\mathrm{L}$ ' in column ' $\mathrm{H} \Rightarrow \mathrm{V}$ ' indicates which hand a subject had used to report a perceived reversal from horizontal to vertical motion.

revealed no significant RT differences between subjects $[F(9,9)=4.09, P=0.29]$. Furthermore, we found no correlation between RT (averaged across both hands from session 3) and reversal rate (Pearson's $r=0.06$ ), which is consistent with a recent study [46] employing a similar version of the ambiguous motion display as used in the present study.

\subsection{ERFs}

\subsubsection{Steady-state fields}

Oscillatory visual stimulation results in so-called steadystate responses of the visual cortex reflecting the frequency of stimulation [17,60]. Fig. 3 shows the steady-state ERFs from session 4 evoked by the two stimuli A1 and A2 which constitute the ambiguous pattern (cf. Fig. 1). Each of these two stimuli is presented for $165 \mathrm{~ms}$ followed by a $85 \mathrm{~ms}$ blank screen. Accordingly, the presentation rate of the display is $4 \mathrm{~Hz}$-leading to a $4 \mathrm{~Hz}$ steady-state response.

\subsubsection{Comparison of sessions 2 and 3}

Fig. 4 shows the stimulus-locked ERFs in response to exogenous changes of motion direction for sessions 2 and 3 , i.e., without and with a button press, respectively. A P300-like peak occurs shortly after $500 \mathrm{~ms}$ with similar latency and wave form for both the silent-counting and the button-pressing response. An ANOVA over the RMS values for the time interval around this peak (500-550 ms) with the factors response-type (count, button), topography (anterior, posterior) and hemisphere (left, right) yielded a significant interaction of factors topography and responsetype $[F(1,9)=5.16, P<0.05]$. Post-hoc comparisons revealed higher RMS values in response to button presses as compared to silent-counting in anterior regions $[F(1,9)=$ 6.41, $P<0.05$ ], indicating that motor activity related to the button press enhances this component. However, the striking similarity of this component between sessions 


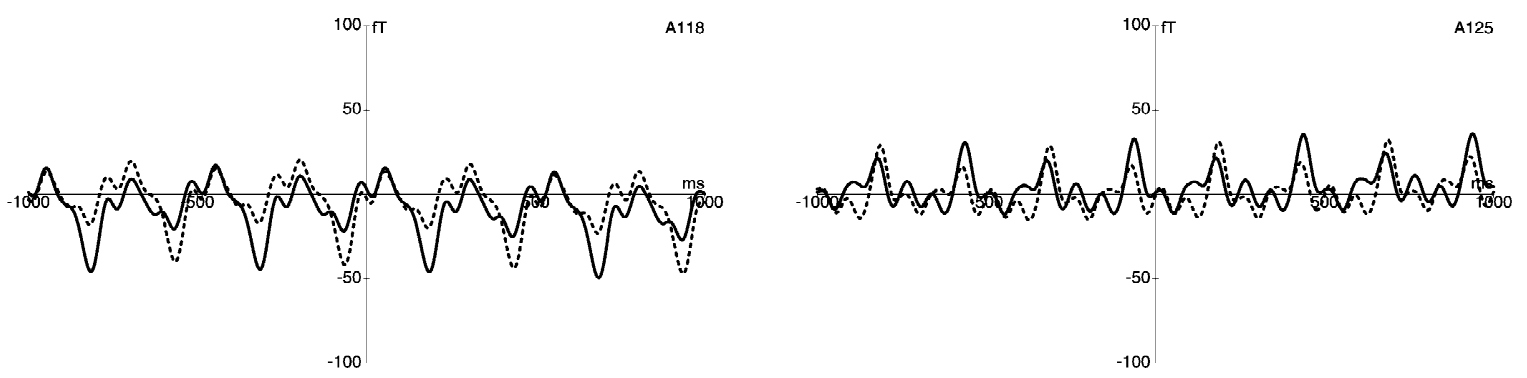

Fig. 3. Grand average ERFs in response to the two stimuli A1 (dotted) and A2 (solid) which constitute the ambiguous pattern. The sensors are located over left (A118) and right (A125) occipital cortex.
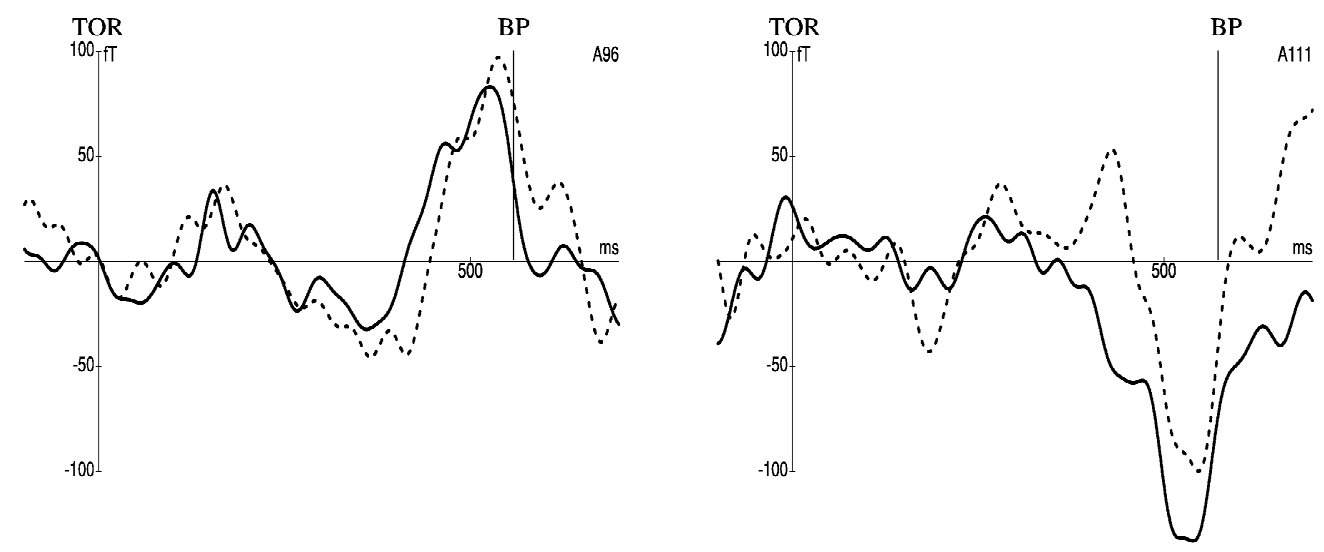

Fig. 4. Grand average ERFs in response to exogenous change of motion direction for silent-counting in session 2 (dotted) and button-pressing in session 3 (solid) for two anterior sensors over the left (A96) and right (A111) hemisphere (stimulus-locked averages). The vertical line (BP) indicates the button-press as estimated from the overall mean RT of $550 \mathrm{~ms}$. Time of reversal (TOR) is at $0 \mathrm{~ms}$.

(especially at sensor A96) in relation to the button-press (indicated by the vertical line in Fig. 4) indicates that premotor activity did not contribute substantially to the exogenous pattern reversal-related component in session 3 .

\subsubsection{Comparison of sessions 3 and 4}

Fig. 5 shows the response-locked grand average ERFs for endogenous and exogenous reversals of motion direc- tion with the estimated time of reversal (indicated by the vertical line). For session 3, the P300-like peak $500 \mathrm{~ms}$ after the exogenous reversal in Fig. 4 corresponds to the peak at approximately $150 \mathrm{~ms}$ prior to the button response in Fig. 5 (button press is at $0 \mathrm{~ms}$ in Fig. 5). However, there is a remarkably different morphology of the component following the exogenous pattern reversal dependent on whether the averaging was stimulus-locked or response-
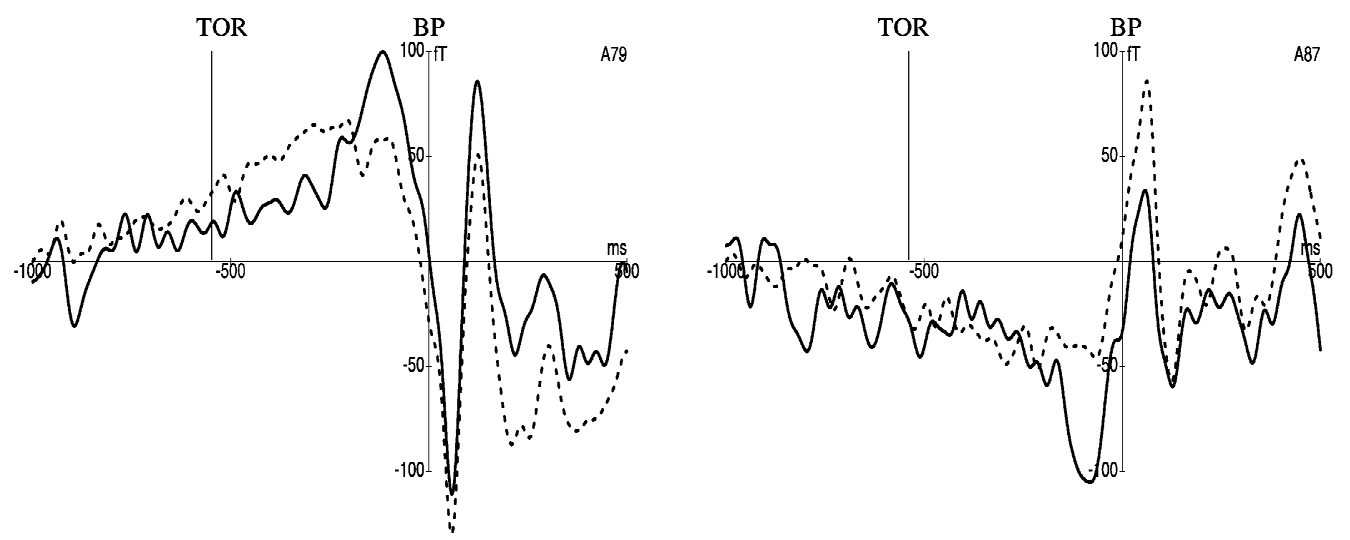

Fig. 5. Grand average ERFs in response to endogenous (session 4, dotted) and exogenous (session 3, solid) changes of motion direction. The sensors over the left (A79) and right (A87) hemisphere show a slow potential preceding the button-press response (BP at 0 ms; response-locked averages). The vertical line (TOR) indicates the time of reversal as estimated from the mean RT of exogenous pattern reversal obtained in session 3 . 


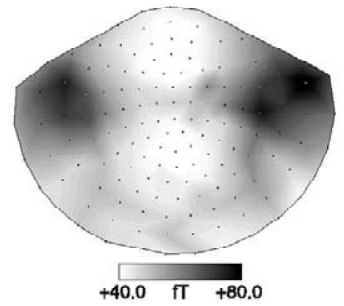

exogenous

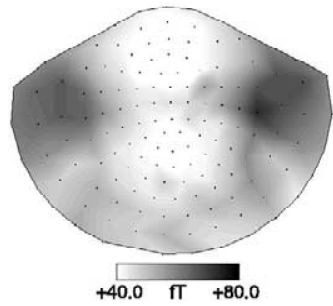

endogenous
Fig. 6. Topographical distribution of the grand average total gamma activity in the time interval -1000 to $-100 \mathrm{~ms}$ before the response (without baseline correction).

locked, indicating an increased jitter of the perceptual reversal-related activity when averaging backwards from the motor response. The endogenous reversal-related component in Fig. 5 shows a morphology similar to that of the exogenously generated component, although a clear peak is missing. Therefore, an ANOVA of the RMS values in the time interval around the peak of the exogenous component (from 200 to $100 \mathrm{~ms}$ before the push-button response) was performed with factors reversal-type (exogenous, endogenous), topography (anterior, posterior), and hemisphere (left, right). A significant main effect of reversal-type revealed larger RMS values for exogenous (68 fT) as compared to endogenous (50 fT) responses $[F(1,9)=8.17, P<0.05]$. Furthermore, a significant interaction of reversal-type $\times$ hemisphere $[F(1,9)=6.43, P<$ 0.05 ] indicated that within the time interval from -200 to $-100 \mathrm{~ms}$ responses to endogenous reversals were larger over the left ( $58 \mathrm{fT}$ ) than over the right hemisphere (42 fT), while this was not the case for responses to exogenous reversals (left: $67 \mathrm{fT}$, right: $68 \mathrm{fT}$ ).

\subsection{Oscillatory activity}

\subsection{1. $40 \mathrm{~Hz}$ (gamma)}

Fig. 6 shows the topographical distribution of the total gamma activity for endogenous and exogenous pattern reversals. The generators for gamma activity in response to endogenous and exogenous pattern reversals turned out to be very similar, since the topographical distribution was much the same. An ANOVA was performed for the total gamma activity with factors reversal-type (exogenous, endogenous), topography (anterior, posterior), hemisphere (left, right), and time window (10 time intervals of $100 \mathrm{~ms}$ each preceding the button press). According to the estimated time of reversal, binding-related gamma activity was expected for the time interval around $500 \mathrm{~ms}$ before the button press. However, the ANOVA did not yield any significant effects.

\subsection{2. $10 \mathrm{~Hz}$ (alpha)}

Fig. 7 shows the topographical distribution of the total alpha activity for endogenous and exogenous changes of motion direction. A clear difference between the alpha activity in the exogenous and endogenous conditions can be seen in both amplitude and topography.

Fig. 8 shows the time course of the total alpha activity for exogenous and endogenous changes of motion direction relative to the button press. As can be seen, activity
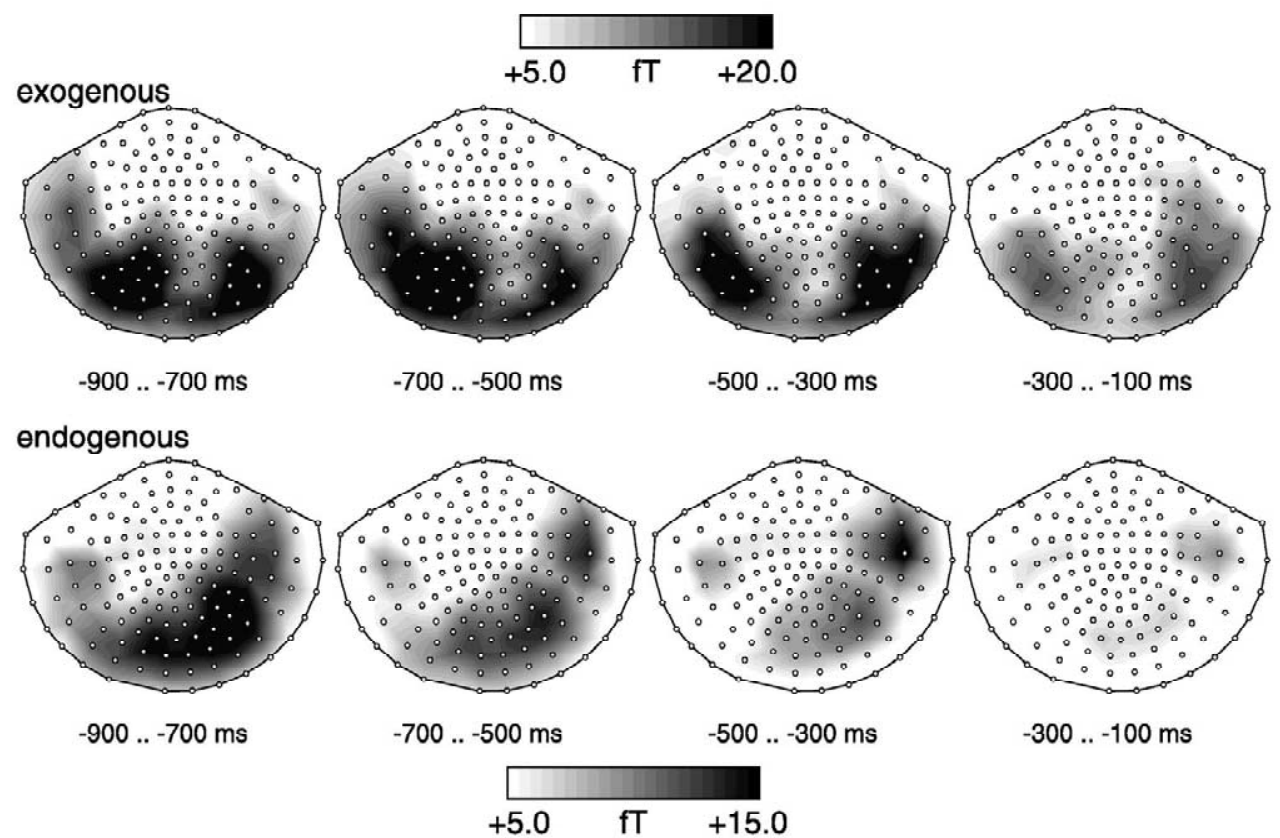

Fig. 7. Topographical distribution of the grand average total alpha activity before the response in sessions 3 (exogenous, top) and session 4 (endogenous, bottom) with baseline correction for the time interval 0-50 ms after the button press. A clear difference between the alpha activity in the exogenous and endogenous conditions can be seen. 

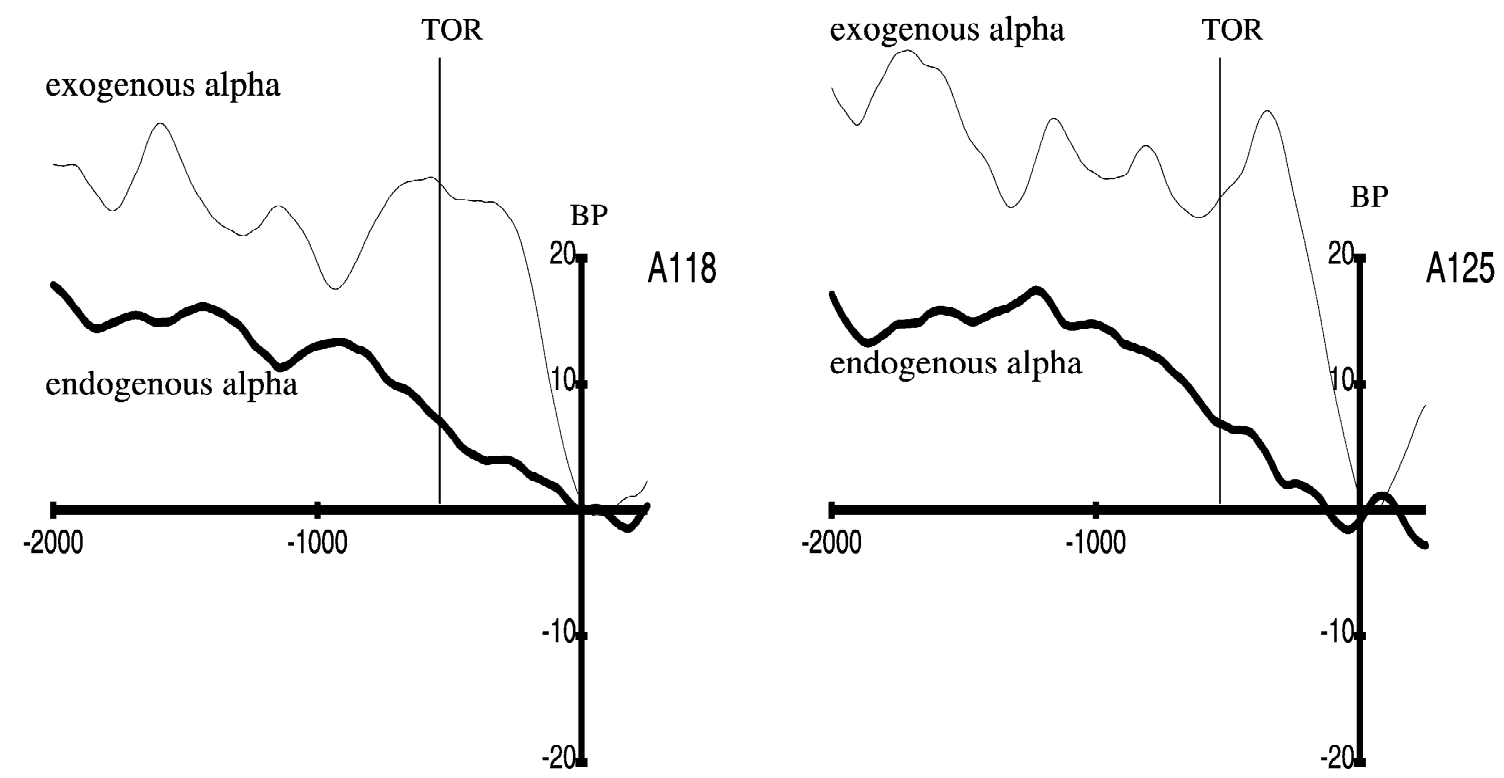

Fig. 8. Grand average response-related total alpha for exogenous (session 3, thin line) and endogenous (session 4, thick line) changes of perceived motion direction for two posterior sensors over left (A118) and right (A125) hemisphere. Button press (BP) is at 0 ms. Baselines in the time interval of button pressing $(0-50 \mathrm{~ms})$ were subtracted for visualization. The vertical line (TOR) indicates the estimated time of reversal.

for the exogenous pattern reversal is increased. Of more direct interest for the present work, the time course of the alpha activity preceding the estimated time of reversal (as indicated by the vertical line) differs between conditions, such that the alpha time course for the exogenous condition oscillates around a relatively constant value until it drops suddenly at the estimated time point of reversal, while the endogenous alpha activity continuously decreases. In order to examine these differences in the time courses statistically, we performed post-hoc time series analyses of the alpha activity ${ }^{1}$. Regression lines were fitted to the data via linear regression and the mean squared error (mse) was computed as an indicator of the goodness-of-fit. Alpha activity was summed across all posterior sensors, as determined from Fig. 7. In a first step, regression lines were fitted to the complete time interval of $-1000-0 \mathrm{~ms}$ in one step. This resulted in a good fit $(\mathrm{mse}=0.35 \mathrm{fT})$ for the endogenous condition (solid line in top panel of Fig. 9), suggesting one underlying process which decays over time. However, for the exogenous condition (dotted line in top panel of Fig. 9) this resulted in a bad fit of the data $(\mathrm{mse}=9.24 \mathrm{fT})$. One underlying process seems not to be sufficient to model the data-but two processes might be appropriate. Therefore, in a second step, we tried to fit two regression lines to the alpha activity of the exogenous condition (bottom panel of Fig. 9). The time point at which we separated the data for separate fits was chosen according to the RT data from the exogenous condition. We assumed that a first process would remain stable until the

\footnotetext{
${ }^{1}$ An anonymous reviewer suggested this method instead of comparing alpha between exogenous and endogenous conditions by means of an ANOVA.
}

change of stimulation was perceived. The ERF data (cf. Fig. 4) suggests that visual processing of the change of stimulation is completed after approximately $250 \mathrm{~ms}$ (return to baseline of the ERFs). Thus, we chose this point in time for the beginning of a hypothetical second process lasting the remaining $300 \mathrm{~ms}$ until the button press. (However, the exact choice of the point of time at which a new process starts is not critical, as can be seen from the following results.) Fitting two regression lines into the alpha activity of the exogenous condition resulted in a much better goodness-of-fit (mse $=0.65 \mathrm{fT}$ ), suggesting that, indeed, two processes are responsible for the time course of the alpha activity (bottom panel of Fig. 9).

\section{Discussion}

\subsection{Behavioral results}

The rate of reversals for ambiguous patterns has been reported to vary substantially across subjects $[9,46,62,63]$, which was also found in the present study. However, the individual reversal rates for each subject remained relatively constant between sessions 1 and 4, which demonstrates that the reversal rate is a reliable measure [6] as long as the subjects are not instructed to control the reversal rate voluntarily [66]. The overall mean reversal rate obtained in the present study ( 8.9 reversals/min) corresponds to the mean reversal rate of so-called high-rate switchers $(8.2$ reversals/min vs. 4.1 reversals/min for the low-rate switchers) reported in a former study using a similar version of the ambiguous motion display [62]. Lower rates of 3 reversals/min were observed in a study 

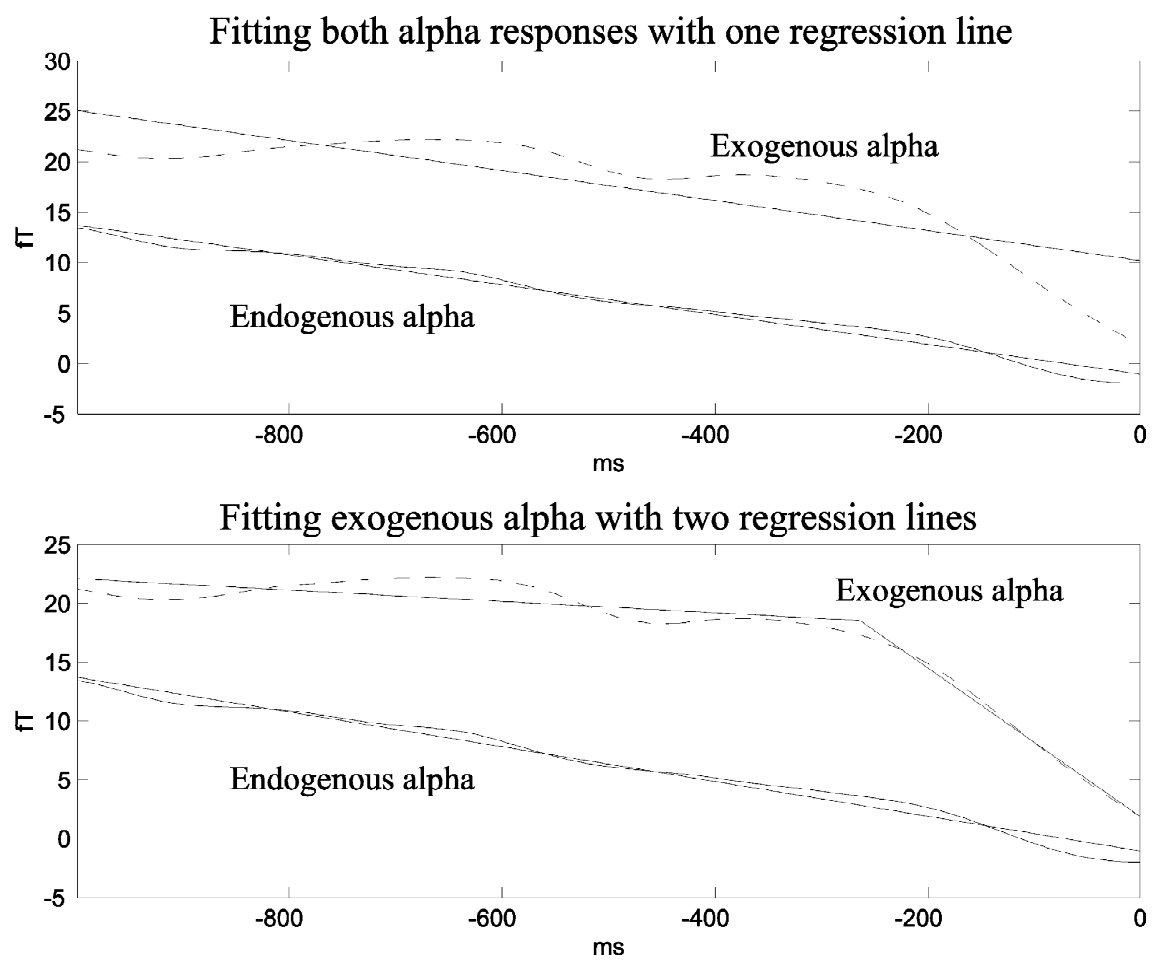

Fig. 9. Linear regression fits of the total alpha activity for the endogenous (solid) and exogenous condition (dotted). Fitting both condition with one regression line (top) results in a bad fit for the exogenous condition. Fitting two regression lines for the exogenous condition, however, yields good fits for both conditions (bottom).

employing the ambiguous motion display with a $1 \mathrm{~Hz}$ alternation frequency of the diagonal pairs of dots which constitute the ambiguous motion [46]. This slowing of the reversal rate can be explained by the low repetition rate (1 $\mathrm{Hz}$ ), since it is known that the reversal rate obtained with the ambiguous motion display increases with increasing alternation frequency of the diagonal pairs of dots [35].

Error rates were higher for the silent-counting (5.6\%) than button-pressing (3.2\%) task, indicating that maintaining the accurate count is more demanding than preparation and execution of motor responses in the button-pressing task. In general, the mean error rates of both response types are comparable to error rates found with other visual discrimination tasks $[18,19,65]$. In session 3 , no significant RT differences between hand of response and between subjects were obtained, indicating that task difficulty was similar for all subjects, and that RTs with the dominant (right) hand were not significantly shorter than with the non-dominant (left) hand. In fact, half of the subjects responded even slightly faster with their left hand. This finding is not congruent with the general observation of longer RTs for the non-dominant hand [56]. This inconsistency may be explained by a response strategy of the subjects favoring accuracy of the response at the cost of maximum speed, such that responding as accurately as possible slowed down the RT to a level at which the usual RT difference between dominant and non-dominant hands disappeared. However, the overall mean RT of $550 \mathrm{~ms}$ is congruent to RTs obtained with dextral button-presses in conventional oddball visual tasks [65]. In sum, although the button-pressing response was easier than the silentcounting task in the exogenous sessions, these behavioral findings suggest that performance and accuracy were high across all experimental conditions.

\subsection{ERFs}

In the present study, button-press responses were inevitably required in order to signal the endogenous reversals occurring with the ambiguous motion display. In addition, the averaging had to be performed responselocked to the button-press as reference, because of a lacking external event in multistable perception. Voluntary movements causes the occurrence of distinct movementrelated potentials (MRPs) in EEG and MEG recordings $[32,34]$. This introduces the possibility of an overlap between stimulus-locked cognitive potentials like the P300 and MRP components in tasks that require button-presses, especially in conditions when the motor response is coincident with or close to the peak of P300 [30]. Therefore, we had to examine the influence of responselocked averaged MRPs on the slow component following the ambiguous pattern reversal. This was performed by a stepwise comparison of the exogenous and endogenous pattern reversal conditions.

Firstly, we showed that the contribution of MRPs to 
P300-like components following exogenously generated pattern reversals with conventional stimulus-locked averaging is not substantial. This could be inferred from the similar latency and morphology of the ERFs elicited in session 2 (without motor response) and session 3 (with motor response). Secondly, a comparison of the same exogenously generated component (session 3 ) between stimulus-locked and response-locked averaging revealed a remarkable difference of the resulting wave forms. Since the contribution of MRPs is the same, this difference in wave forms indicate a 'smearing out' of the cognitive components due to RT variability which results in increased jittering when averaging response locked (backwards) as compared to averaging stimulus-locked (forwards). Accordingly, we argue that also the morphology of the slow component in the ambiguous pattern reversal condition is caused by increased jitter due to RT variability which is inherent in response-locked averaging and not caused solely by MRPs that are related to the preparation of voluntary movements. Thus, it can be concluded that this component reflects activity that is related to the processing of endogenous perceptual reversals as it was shown previously in EEG experiments [5,22,47]. The occurrence of the slow component in response to both endogenous and exogenous pattern reversals probably indicates that the conscious recognition of a perceptual change is processed similarly for endogenous and stimulus-driven reversals. However, the amplitude of the slow component was reduced for the endogenous reversal in comparison to the exogenous pattern change which is consistent with EEG findings [64]. This amplitude reduction can be related to 'equivocation' or post-hoc uncertainty due to a higher discrimination difficulty for the endogenous pattern change, as known from the P300 literature $[24,31]$.

\subsection{Oscillatory activity}

\subsection{1. $40 \mathrm{~Hz}$ (gamma)}

Even though gamma activity changes in relation to spontaneous perceptual reversals were found in previous EEG experiments on multistable perception [4], our MEG results did not show significant differences of gamma activity between exogenous and endogenous change of motion direction. According to our a priori hypothesis about binding-related gamma activity, we did not expect differences between the endogenous and exogenous pattern reversals, which is congruent with the nearly identical topographical maps of the gamma activity for the two conditions. However, we expected an increase of gamma activity for both the endogenous and exogenous pattern reversals at the time of reversals indicating the binding of the other perceptual alternative. Such a gamma-band increase was not found. Since binding-related gamma activity was found in a number of studies, the failure of this activity in the present study may be due to the small size of the stimuli and the relatively small number of epochs which is limited naturally by the reversal rates of the subjects.

\subsection{2. $10 \mathrm{~Hz}$ (alpha)}

Alpha activity in the human EEG can be classified into different types [3]. Most frequently, alpha activity is found as a spontaneous rhythm [50]. However, alpha activity can also be evoked by experimental stimuli and reflects cognitive processes $[1,2,28]$. In addition, induced alpha activity, which occurs when alpha desynchronization is not time-locked to an exogenous event, as it is the case in the endogenous condition of the present study, can still be measured [49]. The total alpha activity reported in this study comprised evoked and induced alpha activity. Thus, even the endogenous condition should contribute to this measure.

The marking difference between the total alpha activity in the endogenous and the exogenous condition was not only reflected in different amplitudes and topographies, but also in different time courses. While the endogenous alpha activity constantly decreased over the complete interval preceding the button press, in the exogenous condition the activity fluctuated around a stable level until a sharp decline at about $300 \mathrm{~ms}$ before the button press. This indicates that the exogenous alpha activity decreases in response to the externally induced pattern reversal, therefore reflecting a stimulus-driven process, known as eventrelated desynchronization [51]. Different from that, the decrease of the endogenous alpha activity started already in the time interval before the estimated time point of reversal. Such a time course is more consistent with the assumption of a threshold value that has to be reached before a reversal can occur. These different time courses of the endogenous and exogenous alpha activities are in good accordance with our second hypothesis of a neurophysiological mechanism that reflects the destabilization of the actual percept which is specific to an endogenous self-generating process. Based on this interpretation of the alpha findings, we propose a potential neurophysiological mechanism which initiates endogenous reversals during multistable perception in contrast to externally induced pattern reversals as illustrated in Fig. 9. Our model assumes that the observed alpha activity reflects visual awareness of the perceived motion direction [58]. During endogenous reversals, the representation of the perceived motion direction slowly decays until a threshold is passed and then the alternative motion direction becomes visually aware. In contrast, during exogenous reversals the perceived motion direction is constantly reinforced by external unambiguous stimulation and does not change until the stimulation actually changes. Obviously, this proposed mechanism is congruent with a bottom-up approach to the explanation of reversible figures $[21,29,35,43,72]$, since the slowly decreasing alpha activity probably reflects a passive and automatic process which is not represented in 
visual awareness. However, a consequence of this underlying process is a sudden change in visual awareness (perceptual reversal), which is reflected by the occurrence of a P300-like component similar to stimulus evaluation processes following 'real' changes in the environment. Proposing this kind of mechanism does, of course, not mean that we deny the importance of top-down influences to figural reversals. There is strong neurophysiological evidence for the impact of top-down processes on the perception of ambiguous figures suggesting that spontaneous alternations reflect responses to active programmed events initiated by brain areas that integrate sensory and non-sensory information to coordinate a diversity of behaviors [39]. An fMRI study, mentioned in the introduction, presented evidence for an intimate linkage of perceptual and attentional processes in the dynamics of metastable visual awareness [27]. Recently, it was shown in EEG experiments that gamma activity plays a role also in attentional top-down processing during multistable perception $[62,63]$. In addition, it has been demonstrated in psychophysical studies that the reversal rate can be influenced by instructions supporting the notion of top-down involvement [66]. Therefore, we assume that a hybrid model comprising both bottom-up and top-down processes could best account for all the observed phenomena. Future research is needed in order to propose an integrative neurophysiological model incorporating spatial and temporal aspects of the interaction between bottom-up and topdown processing in multistable perception.

\section{References}

[1] E. Başar, C. Başar-Eroglu, S. Karakaş, M. Schurmann, Oscillatory brain theory: a new trend in neuroscience, IEEE Eng. Med. Biol. 18 (1999) 56-66.

[2] E. Başar, C. Başar-Eroglu, S. Karakaş, M. Schurmann, Brain oscillations in perception and memory, Int. J. Psychophysiol. 35 (2000) 95-124.

[3] E. Başar, M. Schurmann, C. Başar-Eroglu, S. Karakaş, Alpha oscillations in brain functioning: an integrative theory, Int. J. Psychophysiol. 26 (1997) 5-29.

[4] C. Başar-Eroglu, D. Strüber, P. Kruse, E. Başar, M. Stadler, Frontal gamma-band enhancement during multistable visual perception, Int. J. Psychophysiol. 24 (1996) 113-125.

[5] C. Başar-Eroglu, D. Strüber, M. Stadler, P. Kruse, E. Başar, Multistable visual perception induces a slow positive EEG wave, Int. J. Neurosci. 73 (1993) 139-151.

[6] J.E. Bergum, B.O. Bergum, Reliability of reversal rates as a measure of perceptual stability, Percept. Mot. Skills 50 (1980) 1039-1046.

[7] R. Blake, N.K. Logothetis, Visual competition, Nat. Rev. Neurosci. 3 (2002) 13-21.

[8] Y. Bonneh, D. Sagi, A. Karni, A transition between eye and object rivalry determined by stimulus coherence, Vision Res. 41 (2001) 981-989.

[9] A. Borsellino, A. De Marco, A. Allazetta, S. Rinesi, B. Bartolini, Reversal time distribution in the perception of visual ambiguous stimuli, Kybernetik 10 (1972) 139-144.

[10] R.J. Brown, A method for investigating binocular rivalry real-time with a steady-state VEP, Vision Res. 37 (1997) 2401-2408.
[11] F. Crick, C. Koch, Towards a neurobiological theory of consciousness, Semin. Neurosci. 2 (1990) 263-275.

[12] C.C. Duncan-Johnson, E. Donchin, On quantifying surprise: the variation of event-related potentials with subjective probability, Psychophysiology 14 (1977) 456-467.

[13] P.G. Fitzgerald, T.W. Picton, Event-related potentials recorded during the discrimination of improbable stimuli, Biol. Psychol. 17 (1983) 241-276.

[14] P. Fries, P.R. Roelfsema, A.K. Engel, P. König, W. Singer, Synchronization of oscillatory responses in visual cortex correlates with perception in interocular rivalry, Proc. Natl. Acad. Sci. USA 94 (1997) 12699-12704.

[15] S. Geisser, S. Greenhouse, On methods in the analysis of profile data, Psychometrika 24 (1959) 95-112.

[16] S. He, W.L. Davis, Filling-in at the natural blind spot contributes to binocular rivalry, Vision Res. 41 (2001) 835-840.

[17] C.S. Herrmann, Human EEG responses to $1-100 \mathrm{~Hz}$ flicker: resonance phenomena in visual cortex and their potential correlation to cognitive phenomena, Exp. Brain Res. 137 (2001) 345-353.

[18] C.S. Herrmann, A. Mecklinger, Magnetoencephalographic responses to illusory figures: early evoked gamma is affected by processing of stimulus features, Int. J. Psychophysiol. 38 (2000) 265-281.

[19] C.S. Herrmann, A. Mecklinger, Gamma activity in human EEG is related to high-speed memory comparisons during object selective attention, Vis. Cognit. 8 (2001) 593-608.

[20] C.S. Herrmann, A. Mecklinger, E. Pfeifer, Gamma responses and ERPs in a visual classification task, Clin. Neurophysiol. 110 (1999) 636-642.

[21] J. Hochberg, Figure-ground reversal as a function of visual satiation, J. Exp. Psychol. Hum. Percept. Perform. 19 (1950) 682-686.

[22] U. Isoglu-Alkaç, C. Başar-Eroglu, A. Ademoglu, T. Demiralp, M. Miener, M. Stadler, Analysis of the electroencephalographic activity during the Necker cube reversals by means of the wavelet transform, Biol. Cybern. 79 (1998) 437-442.

[23] U. Isoglu-Alkaç, C. Başar-Eroglu, A. Ademoglu, T. Demiralp, M. Miener, M. Stadler, Alpha activity decreases during the perception of Necker cube reversals: an application of wavelet transform, Biol. Cybern. 82 (2000) 313-320.

[24] R.J. Johnson, A triarchic model of p300 amplitude, Psychophysiology 23 (1986) 367-384.

[25] C. Kaernbach, E. Schröger, T. Jacobsen, U. Roeber, Effects of consciousness on human brain waves following binocular rivalry, Neuroreport 10 (1999) 713-716.

[26] A. Keil, T. Gruber, M.M. Müller, Functional correlates of macroscopic high-frequency brain activity in the human visual system, Neurosci. Biobehav. Rev. 25 (2001) 527-534.

[27] A. Kleinschmidt, C. Buchel, S. Zeki, R.S.J. Frackowiak, Human brain activity during spontaneously reversing perception of ambiguous figures, Proc. R. Soc. Lond. B 265 (1998) 2427-2433.

[28] W. Klimesch, EEG alpha and theta oscillations reflect cognitive and memory performance: a review and analysis, Brain Res. Rev. 29 (1999) 169-195.

[29] W. Kohler, Dynamics in Psychology, Liveright, New York, 1940.

[30] A. Kok, Overlap between P300 and movement-related potentials: a response to Verleger, Biol. Psychol. 27 (1988) 51-58.

[31] A. Kok, H. Looren de Jong, Components of the event-related potential following degraded and undegraded visual stimuli, Biol. Psychol. 11 (1980) 117-133.

[32] H.H. Kornhuber, L. Deecke, Hirnpotentialnderungen bei willkrbewegungen und passiven bewegungen des menschen: bereitschaftspotential und reafferente potentiale, Pflügers Arch. Physiol. 248 (1965) 1-17.

[33] I. Kovacs, T.V. Papathomas, M. Jang, A. Feher, When the brain changes its mind: interocular grouping during binocular rivalry, Proc. Natl. Acad. Sci. USA 93 (1996) 15508-15511.

[34] R. Kristeva-Feige, S. Rossi, B. Feige, T. Mergner, C.H. Lücking, P.M. Rossini, The bereitschaftspotential paradigm in investigating 
voluntary movement organization in humans using magnetoencephalography, Brain Res. Prot. 1 (1997) 13-22.

[35] P. Kruse, M. Stadler, T. Wehner, Direction and frequency specific processing in the perception of long-range apparent motion, Vision Res. 26 (1986) 327-335.

[36] S.-H. Lee, R. Blake, Rival ideas about binocular rivalry, Vision Res. 39 (1999) 1447-1454.

[37] S.R. Lehky, J.H.R. Maunsell, No binocular rivalry in the LGN of alert macaque moneys, Vision Res. 36 (1996) 12225-12234.

[38] D.A. Leopold, N.K. Logothetis, Activity changes in early visual cortex reflect monkeys' percepts during binocular rivalry, Nature 379 (1996) 549-553.

[39] D.A. Leopold, N.K. Logothetis, Multistable phenomena: changing views in perception, Trends Cogn. Sci. 3 (1999) 254-264.

[40] N. Logothetis, Object vision and visual awareness, Curr. Opin. Neurobiol. 8 (1998) 536-544.

[41] N.K. Logothetis, D.A. Leopold, D.L. Sheinberg, What is rivalling during binocular rivalry?, Nature 380 (1996) 621-624.

[42] N.K. Logothetis, J.D. Schall, Neural correlates of subjective visual perception, Science 254 (1989) 761-763.

[43] G.M. Long, T.C. Toppino, G.W. Mondin, Prime time: fatigue and set effects in the perception of reversible figures, Percept. Psychophys. 52 (1992) 609-616.

[44] E.D. Lumer, K.J. Friston, G. Rees, Neural correlates of perceptual rivalry in the human brain, Science 280 (1998) 1930-1934.

[45] E.D. Lumer, G. Rees, Covariation of activity in visual and prefrontal cortex associated with subjective visual perception, Proc. Natl. Acad. Sci. USA 96 (1999) 1669-1673.

[46] T.J. Müller, A. Federspiel, A.J. Falgatter, W.K. Strik, EEG signs of vigilance fluctuations preceding perceptual flips in multistable illusionary motion, Neuroreport 10 (1999) 3423-3427.

[47] B.F. O'Donnell, T. Hendler, N.K. Squires, Visual evoked potentials to illusory reversals of the Necker cube, Psychophysiology 25 (1988) 137-143.

[48] B.S. Oken, K.H. Chiappa, Statistical issues concerning computerized analysis of brain-wave topography, Ann. Neurol. 19 (1986) 493494.

[49] G. Pfurtscheller, D. Flotzinger, W. Mohl, M. Peltoranta, Prediction of the side of hand movements from single-trial multi-channel EEG data using neural networks, Electroencephalogr. Clin. Neurophysiol. 82 (1992) 313-315.

[50] G. Pfurtscheller, A. Stancak, C. Neuper, Event-related synchronization (ERS) in the alpha band-an electrophysiological correlate of cortical idling: a review, Int. J. Psychophysiol. 24 (1996) 39-46.

[51] G. Pfurtschller, F.H. Lopes da Silva, Event-related EEG/MEG synchronization and desynchronization: basic principles, Clin. Neurophysiol. 110 (1999) 1842-1857.

[52] J. Polich, T. Bondurant, P300 sequence effects, probability, and interstimulus interval, Physiol. Behav. 61 (1997) 843-849.

[53] A. Polonsky, R. Blake, J. Brown, D.J. Heeger, Neuronal activity in human primary visual cortex correlates with perception during binocular rivalry, Nat. Neurosci. 3 (2000) 1153-1159.

[54] G. Rees, Neuroimaging of visual awareness in patients and normal subjects, Curr. Opin. Neurobiol. 11 (2001) 150-156.

[55] A. Revonsuo, Visual perception and subjective awareness, Behav. Brain Sci. 21 (1998) 796.
[56] D.F. Salisbury, B. Rutherford, M.E. Shenton, R.W. McCarley, Button-pressing affects P300 amplitude and scalp topography, Clin. Neurophysiol. 112 (2001) 1676-1684.

[57] F. Sengpiel, C. Blakemore, R. Harrad, Interocular suppression in the primary visual cortex: a possible neural basis of binocular rivalry, Vision Res. 35 (1995) 179-195.

[58] T.V. Sewards, M.A. Sewards, Alpha-band oscillations in visual cortex: part of the neural correlate of visual awareness?, Int. J. Psychophysiol. 32 (1999) 35-45.

[59] D.L. Sheinberg, N.K. Logothetis, The role of temporal cortical areas in perceptual organization, Proc. Natl. Acad. Sci. USA 94 (1997) $3408-3413$.

[60] R.B. Silberstein, Steady-state visually evoked potentials, brain resonances, and cognitive processes, in: P.L. Nunez (Ed.), Neocortical Dynamics and Human EEG Rhythms, Oxford University Press, Oxford, 1995, pp. 272-303.

[61] R. Srinivasan, D.P. Russell, G.M. Edelman, G. Tononi, Increased synchronization of neuromagnetic responses during conscious perception, J. Neurosci. 19 (1999) 5435-5448.

[62] D. Strüber, C. Başar-Eroglu, E. Hoff, M. Stadler, Reversal-rate dependant differences in the EEG gamma-band during multistable perception, Int. J. Psychophysiol. 38 (2000) 243-252.

[63] D. Strüber, C. Başar-Eroglu, M. Miener, M. Stadler, EEG gammaband response during the perception of Necker cube reversals, Vis. Cognit. 8 (2001) 609-621.

[64] D. Strüber, C. Jacobs, C. Başar-Eroglu, M. Stadler, Visual eventrelated potentials during perceptual switches of ambiguous and umambiguous dynamic motion patterns: single sweep analysis, Psychophysiology 36 (1999) S112.

[65] D. Strüber, J. Polich, P300 and slow wave from oddball and single-stimulus visual tasks: inter-stimulus interval effects, Int. J. Psychophysiol., in press.

[66] D. Strüber, M. Stadler, Differences in top-down influences on the reversal rate of different categories of reversible figures, Perception 28 (1999) 1185-1196.

[67] C. Tallon, O. Bertrand, P. Bouchet, J. Pernier, Gamma-range activity evoked by coherent visual stimuli in humans, Eur. J. Neurosci. 7 (1995) 1285-1291.

[68] C. Tallon-Baudry, O. Bertrand, Oscillatory gamma activity in humans and its role in object representation, Trends Cogn. Sci. 3 (1999) 151-162.

[69] F. Tong, S.A. Engel, Interocular rivalry revealed in the human cortical blind-spot representation, Nature 411 (2001) 195-199.

[70] F. Tong, K. Nakayama, J.T. Vaughan, N. Kanwisher, Binocular rivalry and visual awareness in human extrastriate cortex, Neuron 21 (1998) 753-759.

[71] G. Tononi, R. Srinivasan, D.P. Russell, G.M. Edelman, Investigating neural correlates of conscious perception by frequency-tagged neuromagnetic responses, Proc. Natl. Acad. Sci. USA 95 (1998) $3198-3203$.

[72] T.C. Toppino, G.M. Long, Selective adaptation with reversible figures: don't change that channel, Percept. Psychophys. 42 (1987) $37-48$.

[73] F. Valle-Inclan, S.A. Hackley, C. de Labra, A. Alvarez, Early visual processing during binocular rivalry studied with visual evoked potentials, Neuroreport 10 (1999) 21-25. 